\title{
La traducción al español de diccionarios médicos alemanes en el siglo XIX*
}

\author{
CARMEN QUIJADA DIEZ \\ Universidad de Oviedo \\ quijadacarmen@uniovi.es
}

BERTHA M. GUTIÉRREZ RODILLA

Universidad de Salamanca

bertha@usal.es

\section{INTRODUCCIÓN}

En el presente trabajo se presenta el estudio traductológico preliminar llevado a cabo sobre dos diccionarios médicos alemanes del siglo XIX y traducidos al español al final de la centuria. La pertinencia de este estudio radica en que se trata de las dos únicas obras lexicográficas procedentes del ámbito germano y vertidas al castellano en un momento en que la traducción científica se ejercía sobre textos procedentes casi siempre de Francia (Gutiérrez Rodilla 2009: 235-236), lo que los convierte en una fuente de singular valía para el estudio de la recepción de la ciencia en la España decimonónica. Se trata, por un lado, de la obra de Albert Eulenburg Real-Encyclopädie der gesamten Heilkunde: Medizinisch-chirurgisches Handwörterbuch für praktische Ärzte, que constaba en su primera edición de 15 volúmenes publicados entre 1880 y 1883 (llegó a alcanzar los 27 volúmenes en su tercera edición), y editado en español entre 1885 y 1891 bajo el título Diccionario enciclopédico de medicina y cirugía prácticas, «traducido directamente y arreglado para uso de los médicos españoles» por Isidoro de Miguel y Viguri; y, por otro, del Handbuch der speciellen Pathologie und Therapie de Hugo von Ziemssen, publicado en 17 volúmenes entre 1874 y 1885 y traducido al castellano por Francisco Vallina entre 1887 y 1901 bajo el título Tratado enciclopédico de patología médica y terapéutica.

\section{CONTEXTO}

El siglo XIX fue testigo de una extraordinaria producción de repertorios lexicográficos del ámbito médico en países como Francia, Alemania o Inglaterra y, en menor medida, en España. En los dos primeros triunfaban los diccionarios enciclopédicos, también llamados diccionarios de cosas o encliclopedias, realias o bibliotecas (según exponen Déchambre y Lereboullet en la Introducción de su Diccionario enciclopédico de ciencias médicas [1864-1889]), y, por efecto de la traducción, también en España (Gutiérrez Ro-

* La realización de este trabajo se ha financiado con una «Ayuda a Grupos de Investigación» del Plan Estratégico de Investigación y Transferencia de Conocimiento de la Universidad de Salamanca, 2017-2018, y una ayuda del Plan Propio de Apoyo y Promoción de la Investigación 2018 de la Universidad de Oviedo.

CARMEN QUIJADA DIEZ, BERTHA M. GUTIÉRREZ RODILLA «La traducción al español de diccionarios médicos alemanes en el siglo XIX», Revista de Lexicografia, XXIII (2017), pp. 185-199 ISSN: 1134-4539, e-ISSN: 2603-667. doi: https://doi.org/10.17979/rlex.2017.23.0.4702 
dilla 1999: 34), mientras que en Inglaterra la lexicografía terminológica tuvo un notable éxito, en detrimento de la enciclopédica. En España, por su parte, no se tradujo ningún diccionario terminológico (también conocidos como diccionarios de palabas o léxicos o incluso vocabularios [Gutiérrez Rodilla 1999]), pero sí se elaboraron varios originalmente en castellano, entre los que destaca el celebérrimo de Manuel Hurtado de Mendoza, el primero de nuestros vocabularios terminológicos de medicina, publicado en 1840 bajo el título Vocabulario médico-quirúrgico, o Diccionario de Medicina y Cirugía, que comprende la etimología y definición de todos los términos usados en estas dos ciencias por los autores antiguos y modernos (sobre esta obra, vid. Gutiérrez Rodilla 2012a y 2012b).

Los diccionarios terminológicos médicos no fueron en todo caso los más numerosos de los publicados en nuestro país en el siglo XIX, puesto que se produjeron hasta quince compendios enciclopédicos de temática médica: cuatro redactados originalmente en español y once traducciones, de las cuales nueve procedían de originales franceses y dos son las protagonistas de este análisis: el Manual de patología especial y terapia de Hugo von Ziemssen y el Diccionario de Albert Eulenburg, si bien este último podría considerarse una suerte de híbrido entre ambas categorías, ya que su disposición es la de un diccionario terminológico al uso e incluso incluye en ocasiones el origen etimológico de las voces que contiene, pero el desarrollo de cada lema es más bien de tipo enciclopédico.

El fenómeno del enciclopedismo médico en Europa y España se trata in extenso en Gutiérrez Rodilla (1999). A continuación se analizará la traducción y adaptación de estas obras al español.

\section{SOBRE LAS TRADUCCIONES Y ADAPTACIONES AL ESPAÑOL}

No es baladí recordar la enorme trascendencia de la traducción en la transmisión del conocimiento a lo largo de la historia en general pero de manera muy particular en lo referido a la difusión de la ciencia y del conocimiento científico entre pueblos y civilizaciones: en el caso de la medicina, la traducción ha sido y es en realidad copartícipe de primer orden en la construcción del conocimiento y evolución humanas, gracias a sus trabajos de adaptación, recopilación, síntesis y divulgación de los textos médicos de todos los tiempos, desde la Casa de la Sabiduría bagdadí y los Tibbónidas granadinos hasta la Escuela de Traductores de Toledo, pasando por las traducciones alejandrinas y el constante trasvase de saberes en la cuenca mediterránea (Montalt i Resurrecció 2002 y 2005: 50-51; Gutiérrez Rodilla 2009: 230; Eckart 2009: 63-64 y 72-76; Olohan 2009: 246).

Si centramos la atención ahora únicamente en los diccionarios enciclopédicos médicos que aquí se tratan, se debe tener en cuenta que estas obras incluían información muy extensa de muy diversa índole, pero siempre adaptada a los lectores de los países en que se habían publicado inicialmente, de ahí que los traductores médicos que se ocuparon de trasladar y adaptar estas obras a otras lenguas se enfrentaran a retos de difícil solución, pues debían en ocasiones traicionar la fidelidad al contenido del texto original o incluso adaptar los textos a la moral vigente en la España decimonónica (Gutiérrez Rodilla y Quijada Diez 2015: 202). Así se recoge, de hecho, en los títulos de algunas de las obras traducidas: «traducida y adicionada, arreglada a nuestro clima y costumbres», o, como es el caso de la traducción al español del trabajo de Eulenburg, «traducido directamente y arreglado para uso de los médicos españoles». 
En la traducción y adaptación de diccionarios médicos en la España del XIX se observan cuatro tipos de modificaciones respecto a los textos originales ( $\mathrm{vid}$. a este respecto Gutiérrez Rodilla y Quijada Diez 2015):

- Síntesis de contenido: tanto en las obras francesas como en las alemanas el afán de exhaustividad de los autores que intervenían en la empresa, que solían ser varios - incluso muchos - , determinaba que estos diccionarios necesitaran de gran cantidad de volúmenes para poder acoger todo lo que se pretendía incluir en ellos. Sirva como ejemplo el extraordinario crecimiento que experimentaron las sucesivas ediciones del diccionario enciclopédico alemán de Albert Eulenburg, que pasó de tener 15 volúmenes en su primera edición (1880-1883), un número ya bastante elevado, a 22 en la segunda edición (1885-1890) y a nada menos que 27 en su tercera edición (18931901). Sin embargo, la recepción del enciclopedismo en España tuvo que adaptarse al mercado español de la época y a las condiciones económicas del país, de modo que los repertorios enciclopédicos franceses traducidos en España tuvieron que reducir drásticamente el número de volúmenes al verterse al español, como sucedió por ejemplo con el Diccionario de Ciencias Médicas, que pasó de los 60 franceses a los 39 españoles; o el de Adelon, que de los 29 franceses pasó a los 8 españoles. En el caso del diccionario alemán antes citado de Eulenburg, de los 15 volúmenes iniciales de la primera edición de la obra original se pasó a 13 en español. Llamativamente, el manual de Ziemssen pasó de 15 volúmenes originales a 22 en su versión castellana, si bien, como se verá, el aumento del número de volúmenes se debe más bien a una cuestión editorial y comercial que a ampliaciones de contenido.

- Modificación del contenido para actualizarlo. Los procesos editoriales de la época no permitían que el lapso de tiempo transcurrido entre la publicación del original francés o alemán hasta que apareciera su versión en castellano fuera breve, motivo por el que a veces los encargados de esta complementaban algunas entradas añadiendo extractos o información de obras aparecidas con posterioridad a la obra original, y alterando por lo tanto sustancialmente la obra original en su traducción.

- Ampliación o sustitución del contenido para adaptarlo al público destinatario. Un ejemplo revelador en este sentido es el referido a las localidades famosas por la calidad de sus aguas termales o medicinales, como se ejemplificará más adelante.

- Corrección de la información original por errónea o antiespañola. Este comportamiento de los traductores es cuando menos llamativo, como en el caso en que el traductor Federico Borrell, que vertió al castellano la obra francesa Diccionario de los Diccionarios de Medicina, añade dentro de una entrada un listado de autores españoles que se habían ocupado del tema que en modo alguno aparece en el original.

A continuación se exponen algunos datos llamativos que se han observado en el análisis de la versión original y la traducida de ambos diccionarios. Es pertinente realizar un breve inciso respecto al modo en que se ha abordado el estudio de estas obras. Como es bien sabido, los análisis lexicográficos tradicionales suelen basarse en un método de calas cada cierto número de páginas de manera aleatoria, un método que sin duda facilita el hallazgo de material lingüístico digno de estudio. En nuestro caso, al tratarse no solo de un mero análisis lexicográfico de dos obras, sino del estudio con- 
trastivo del texto original y su traducción, y unido ello al hecho de que cada obra consta de un elevado número de volúmenes, se hubo de optar por un abordaje textual de tipo temático. Así, se contrastaron los índices de las obras originales y las traducciones, en una tarea compleja por su envergadura y por la dificultad de acceso a las mismas, para posteriormente centrar la atención en campos de la medicina donde previsiblemente cabría esperar alteraciones en las obras traducidas.

\section{TRADUCCIÓN AL ESPAÑOL DEL DICCIONARIO DE ALBERT EULENBURG}

El título de la obra original es Real-Encyclopädie der gesammten Heilkunde: Mediznisch-chirurgisches Handwörterbuch für praktische Ärzte, y constaba en su primera edición, como se ha dicho, de 15 volúmenes, publicados entre 1880 y 1883.

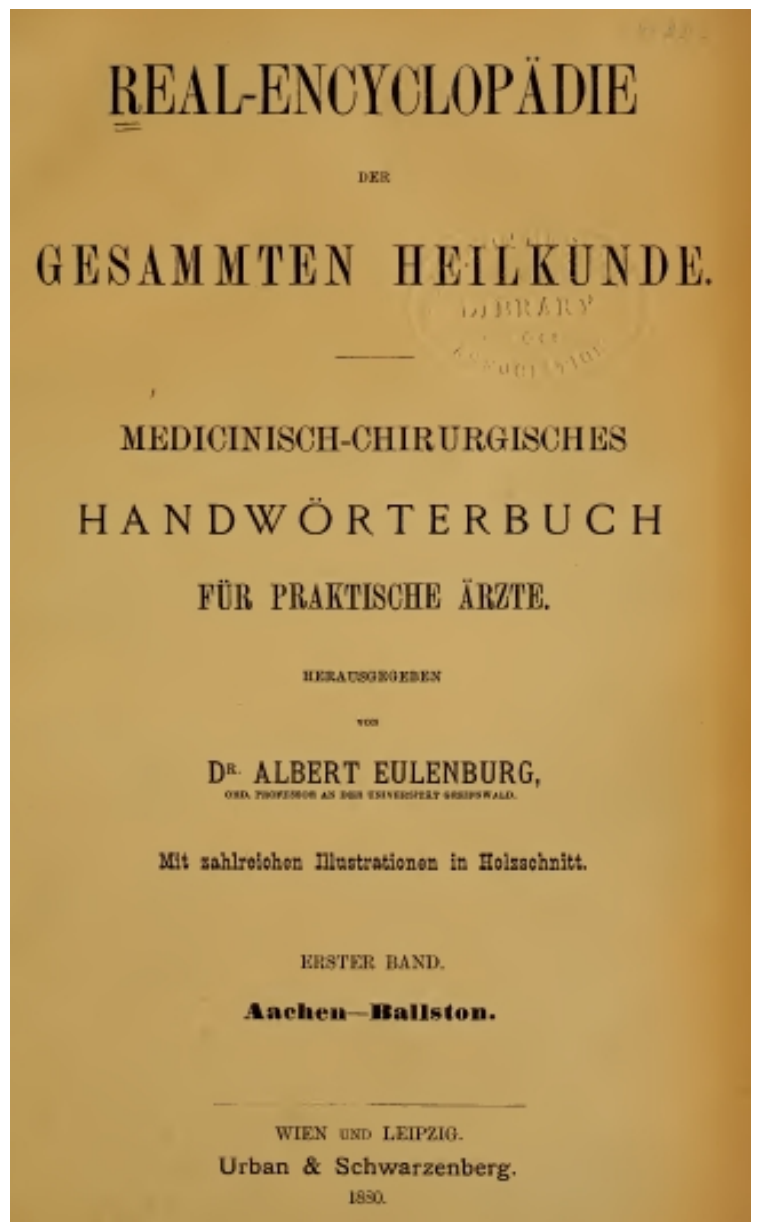

Figura 1: Portada del primer volumen de la edición original en alemán del Diccionario de Euleburg 
La versión en español se publicó entre 1885 y 1891 en 13 volúmenes bajo el título Diccionario enciclopédico de Medicina y Cirugía prácticas, escrito en alemán bajo la dirección del Dr. A. Eulenburg; traducido directamente y arreglado para uso de los médicos españoles por el Dr. D. Isidoro de Miguel y Viguri...; precedido de un prólogo por el Dr. D. Carlos María Cortero. El prólogo que se anuncia en este título no se ha encontrado en ninguno de los ejemplares consultados para este trabajo, lo que permite suponer que nunca llegó a publicarse. La obra la publicó en Madrid el editor Agustín Jubera.

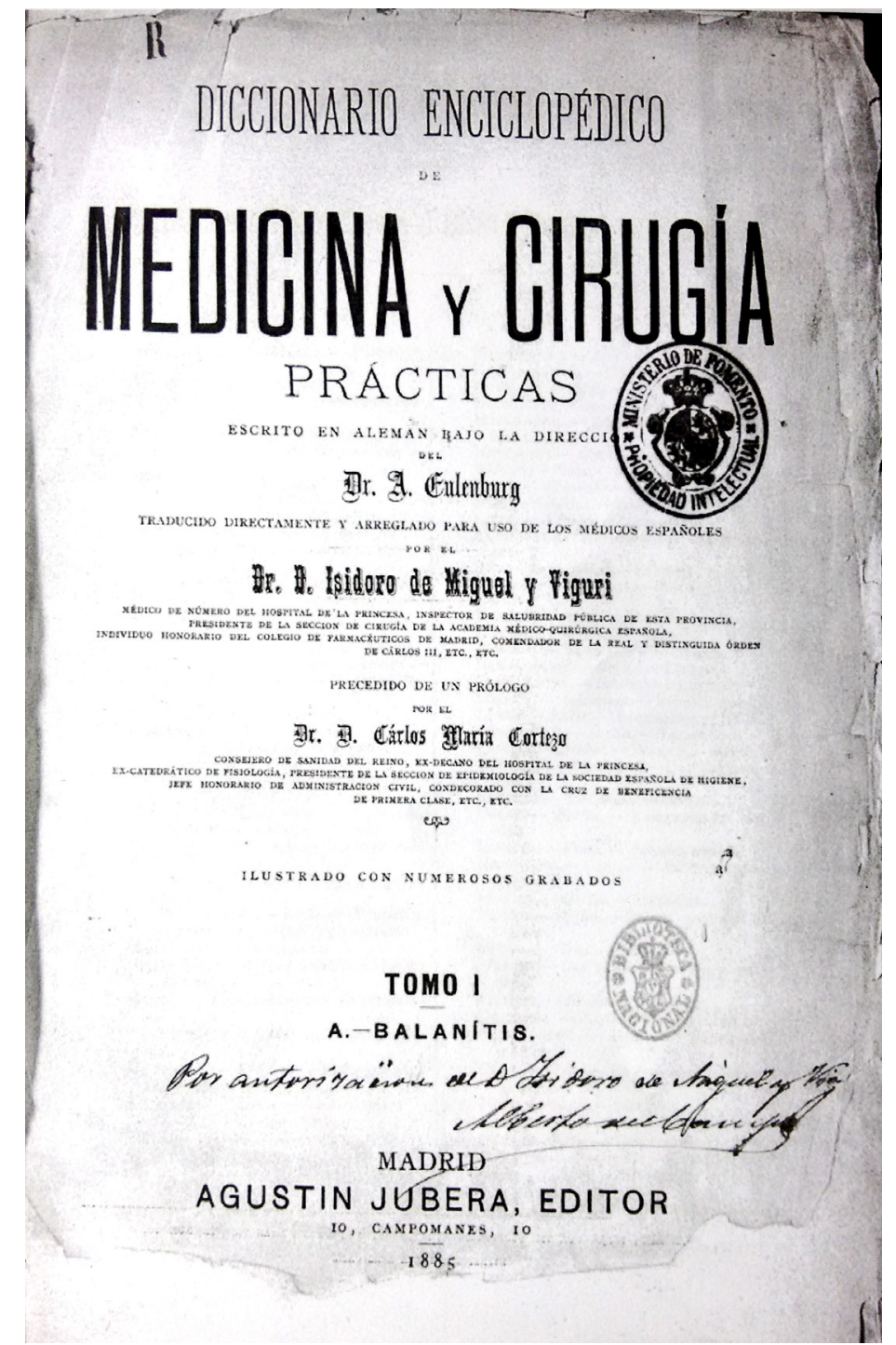

Figura 2: Portada del primer tomo de la edición española del trabajo de Eulenburg. 
En un trabajo anterior (Quijada Diez 2016) ya se reflejaron algunos de los hallazgos del análisis efectuado sobre esta obra, pero es pertinente destacar algún hecho llamativo, como el que nos proporciona el empleo del agua tibia y la caliente como terapias: entre la primera y la cuarta edición originales se produce un cambio sustancial en el empleo de la balneoterapia, de manera que lo que en la primera edición se daba por bueno («los efectos terapéuticos del agua fría en sus diversas formas de aplicación») se desecha por completo en la cuarta («erróneamente se denomina a la hidroterapia cura con agua fría, cuando la temperatura del agua que nos da sensación de calor también encuentra aplicación hidroterapéutica»), es decir, la medicina dio por buenos los baños en aguas tibias y calientes precisamente en el intervalo que va desde la primera edición de este diccionario enciclopédico (1880-1883) a la cuarta (1878-1963). Es un dato que viene a refrendar lo sujeta que se hallaba la labor de los editores de enciclopedias médicas a los avances científicos y lo urgente que era verter con la máxima celeridad los nuevos hallazgos a otras lenguas.

En lo que se refiere a los aspectos de traducción y adaptación antes citados, la búsqueda de materiales se realizó sobre ámbitos de la medicina o lemas concretos en los que cabría pensar que se encontrarían diferencias significativas entre la obra original y la traducida, como el de las aguas medicinales y termales, la acuapuntura, la balneoterapia y la hidroterapia. En alemán se buscó bajo los prefijos Bad-, Wasser- e Hydro-. En español se buscaron similitudes bajo los prefijos hidro-, balneo-, acu- y bajo baños y agua. Hay que decir que, siendo la balneología una de las ramas de la medicina más estudiadas en el ámbito germano, cabría esperar un lema voluminoso bajo los vocablos Balneologie, Balneotherapie o su equivalente en alemán vernáculo Heilbadtherapie. Sin embargo, no existe ninguna entrada para esos vocablos en la obra original, un dato aún más llamativo si cabe cuando detectamos que en la obra en alemán, en la nómina de colaboradores, aparece el doctor E. H. Kirsch, de Marienbad y Praga, para esa rama de la medicina que alemán denominan Balneologie. Tampoco en la versión en español se encuentra lema alguno con el prefijo balneo-.

Al comparar lemas concretos en una versión y otra encontramos ejemplos correspondientes a los cuatro tipos antes citados de traducción y adaptación (ejemplos tratados in extenso en Quijada Diez 2016: 375-379):

\begin{tabular}{|l|l|}
\hline \multicolumn{1}{|c|}{ Versión original } & \multicolumn{1}{c|}{ Versión traducida } \\
\hline Aquapunctur & Lema $100 \%$ fiel al original $s . v$. acuapuntura \\
\hline Bad & Se dan omisiones y reducciones $s$. v. baños \\
\hline Hydrotherapie & Lema $100 \%$ fiel al original $s . v$. hidroterapia \\
\hline Hydrothorax & Lema $100 \%$ fiel al original $s . v$. hidrotórax \\
\hline $\begin{array}{l}\text { Wasser }+ \text { Wasser- } \\
\text { versorgung }\end{array}$ & $\begin{array}{l}\text { Se sintetizan en una única entrada en español }(s . v \text {. agua } \text { ) dos } \\
\text { lemas originales (Wasser y Wasserversorgung), se dan omisiones } \\
\text { (se elimina una tabla con datos sobre el abastecimiento de agua } \\
\text { en ciudades alemanas y austríacas) y reducciones significativas } \\
\text { de contenido. }\end{array}$ \\
\hline
\end{tabular}

Tabla 1: Correspondencia de lemas entre el original y la traducción del Diccionario de Eulenburg 


\section{TRADUCCIÓN AL ESPAÑOL DEL MANUAL DE HUGO VON ZIEMSSEN}

El título de la obra original es Handbuch der speciellen Pathologie und Therapie, y constaba en su primera edición, como se ha dicho, de 17 volúmenes, publicados entre 1874 y 1885 en la ciudad de Leipzig.

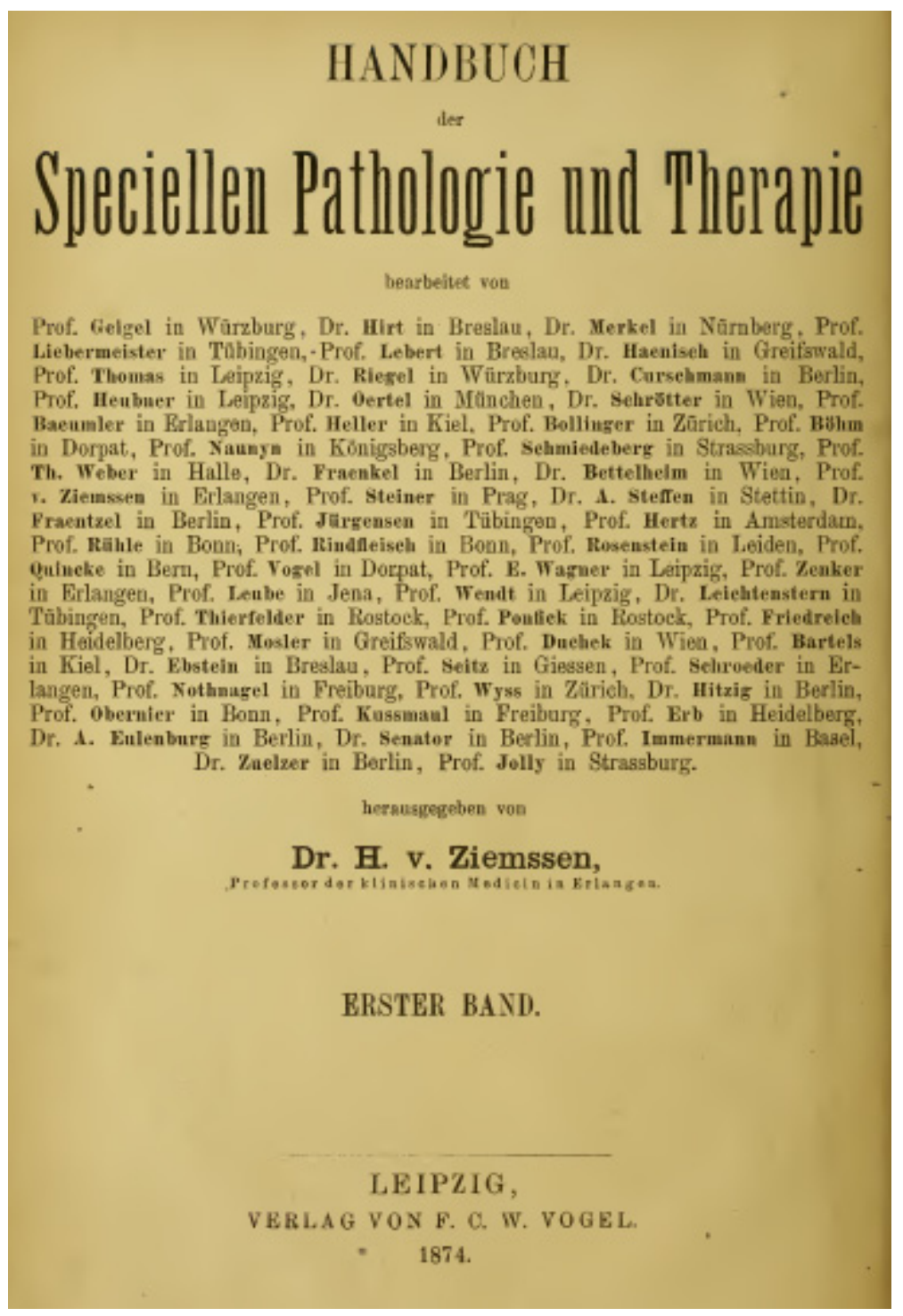

Figura 3: Portada del primer volumen de la edición original en alemán del Handbuch de von Ziemssen 
La versión en español se publicó en Madrid por los Sucesores de Rivadeneyra entre los años 1887 y 1901 en 22 volúmenes bajo el título Tratado enciclopédico de patología médica y terapéutica, publicado bajo la dirección del Dr. H. Ziemssen y traducido al castellano por el Dr. Francisco Vallina.

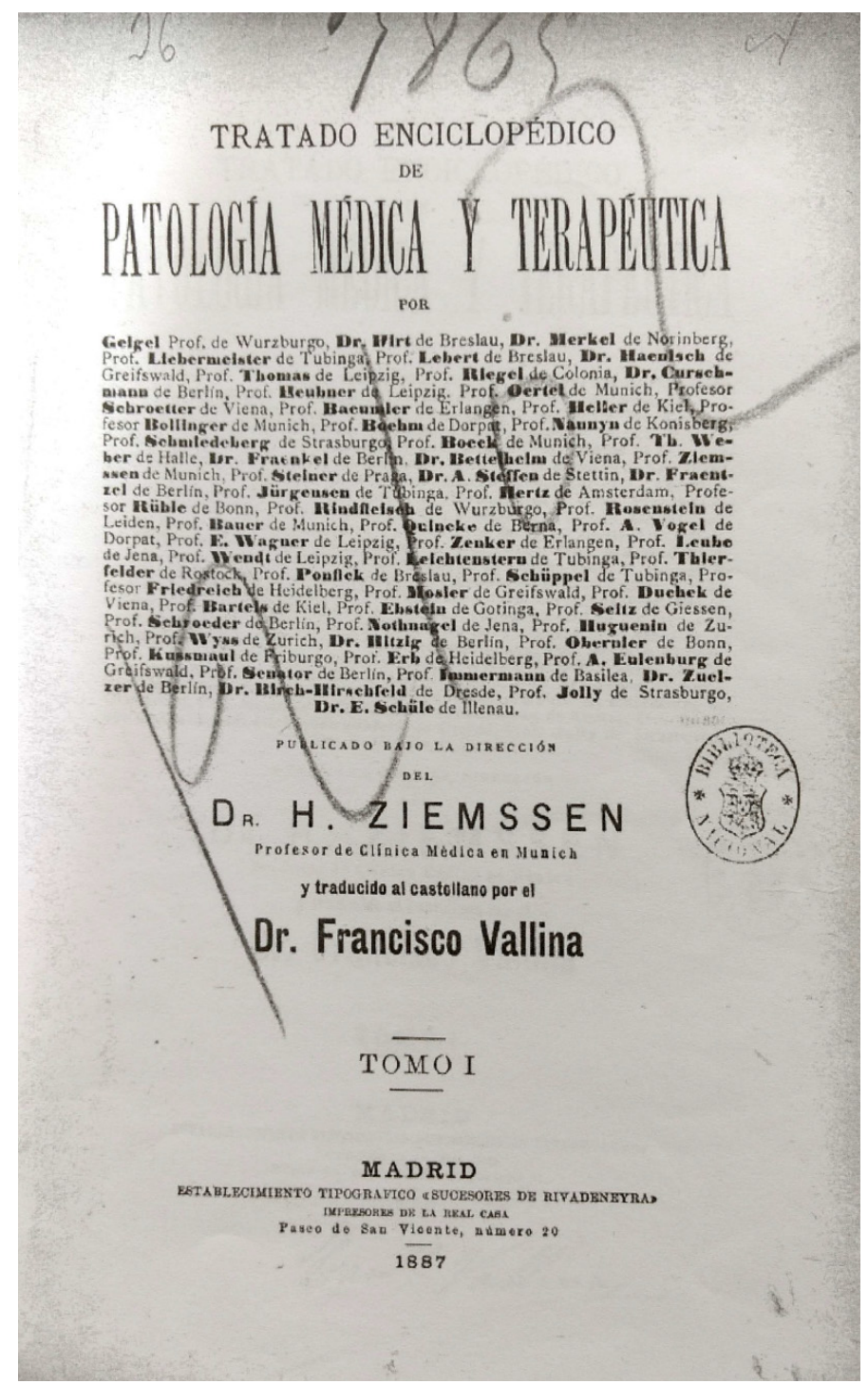

Figura 4: Portada del primer volumen de la traducción al español del Tratado de von Ziemssen 
En las últimas páginas del primer volumen publicado en español aparece la siguiente nota:

Este tomo es el primero de la edición española de la notable obra que con universal aceptación se está publicando actualmente en Alemania por gran número de distinguidos profesores, bajo la dirección del Dr. H. Ziemssen. Del mismo modo que hasta aquí, en lo sucesivo cada mes saldrá á luz un cuaderno de 160 páginas, con las mismas condiciones materiales, al precio de 2,50 pesetas en toda España. El precio de los tomos, una vez terminados, será mayor que por suscripción.

Es una información que permite adivinar el ritmo que se imponían los traductores para la publicación de su trabajo, y de la que también se deduce que el incremento en el número de volúmenes en la traducción (17 en el original, o más bien 16, ya que el tomo 17 es el índice con la nómina de autores y de lemas, frente a los 22 de la traducción) se puede deber más bien a un criterio comercial que de otro tipo, puesto que lo que en la versión alemana ocupa un solo tomo se ve publicado en español en varios, como se verá a continuación.

\subsection{Correspondencias}

De hecho, el primer hallazgo del que podemos dar cuenta está precisamente en la distribución de la información, que varía notablemente de la versión original a la traducida (vid. tabla 2).

\begin{tabular}{|c|c|}
\hline Versión original & Traducción \\
\hline Tomo I. Öffentliche Gesundheits-Pflege & - \\
\hline Tomo II. Acute Infectionskrankheiten & $\begin{array}{l}\text { Tomo XX. Enfermedades infecciosas I. } \\
\text { (1898) }\end{array}$ \\
\hline \multirow{2}{*}{$\begin{array}{l}\text { Tomo III. } \\
\text { tionskrankheiten }\end{array}$} & $\begin{array}{l}\text { Tomo XXI. Enfermedades infecciosas II. } \\
\text { (1899) }\end{array}$ \\
\hline & $\begin{array}{l}\text { Tomo XXII. Enfermedades infecciosas III. } \\
\text { (1901) }\end{array}$ \\
\hline $\begin{array}{l}\text { Tomo IV. Krankheiten } \quad \text { des } \\
\text { Respirationsapparates I. }\end{array}$ & $\begin{array}{l}\text { Tomo I. Enfermedades del aparato } \\
\text { respiratorio I. (1887) }\end{array}$ \\
\hline \multirow{2}{*}{$\begin{array}{l}\text { Tomo V. } \quad \text { Krankheiten } \\
\text { Respirationsapparates II. }\end{array}$} & $\begin{array}{l}\text { Tomo II. Enfermedades del aparato } \\
\text { respiratorio II. (1887) }\end{array}$ \\
\hline & $\begin{array}{l}\text { Tomo III. Enfermedades del aparato } \\
\text { respiratorio III. (1887) }\end{array}$ \\
\hline $\begin{array}{l}\text { Tomo VI. Krankheiten des Circulationsap- } \\
\text { parates. }\end{array}$ & $\begin{array}{l}\text { Tomo XVI. Enfermedades del aparato } \\
\text { circulatorio. (1892) }\end{array}$ \\
\hline \multirow{2}{*}{$\begin{array}{l}\text { Tomo VII. Krankheiten des chy- } \\
\text { lopoëtischen Apparates I. }\end{array}$} & $\begin{array}{l}\text { Tomo X. Enfermedades del aparato } \\
\text { quilopoiético I. (1889) }\end{array}$ \\
\hline & $\begin{array}{l}\text { Tomo XI. Enfermedades del aparato } \\
\text { quilopoiético II. (1889) }\end{array}$ \\
\hline
\end{tabular}




\begin{tabular}{|c|c|}
\hline $\begin{array}{l}\text { Tomo } \quad \text { VIII. } \quad \text { Krankheiten } \\
\text { chylopoëtischen Apparates II. }\end{array}$ & $\begin{array}{l}\text { - Tomo XII. Enfermedades del aparato } \\
\text { quilopoiético III. (1890) } \\
\text { - } \quad \text { Tomo XIII. Enfermedades del aparato } \\
\text { quilopoiético IV. (1890) }\end{array}$ \\
\hline Tomo IX. Krankheiten des Harnapparates. & $\begin{array}{l}\text { Tomo XIV. Enfermedades del aparato } \\
\text { urinario I. (1890) } \\
\text { Tomo XV. Enfermedades del aparato } \\
\text { urinario II. (1891) }\end{array}$ \\
\hline $\begin{array}{l}\text { Tomo X. Krankheiten der weiblichen } \\
\text { Geschlechtsorgane. }\end{array}$ & - \\
\hline $\begin{array}{l}\text { Tomo XI. Krankheiten des Nervensystems } \\
\text { I. }\end{array}$ & $\begin{array}{l}\text { Tomo IV. Enfermedades del sistema } \\
\text { nervioso I. (1887) }\end{array}$ \\
\hline $\begin{array}{l}\text { Tomo XII. Krankheiten } \\
\text { Nervensystems II. }\end{array}$ & $\begin{array}{l}\text { nervioso II. (1887) } \\
\text { nervioso III. (1887) } \\
\text { - Tomo VII. Enfermedades del sistema } \\
\text { nervioso IV. (1887) } \\
\text { Tomo VIII. Enfermedades del sistema } \\
\text { nervioso V. (1888) } \\
\text { Tomo IX. Enfermedades del sistema } \\
\text { nervioso VI. (1888) }\end{array}$ \\
\hline 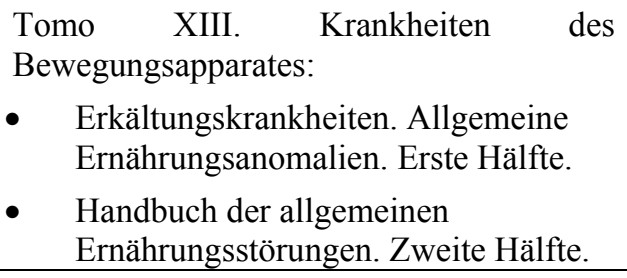 & $\begin{array}{l}\text { - Tomo XVII. Desórdenes generales de } \\
\text { nutrición I. (1894) } \\
\text { Tomo XVIII. Desórdenes generales de } \\
\text { nutrición II. (1895) }\end{array}$ \\
\hline Tomo XIV. Krankheiten der Haut. & - \\
\hline Tomo XV. Intoxicationen. & Tomo XIX. Intoxicaciones. (1896) \\
\hline Tomo XVI. Geisteskrankheiten. & 一 \\
\hline $\begin{array}{l}\text { Tomo XVII. General-Register [Autoren- } \\
\text { Register, Sach-Register] }\end{array}$ & \\
\hline
\end{tabular}

Tabla 2: Correspondencias entre los volúmenes de la edición original y la traducción al castellano del Manual de von Ziemssen

Llama la atención, por ejemplo, la elección del orden en que se publicaron en español los volúmenes: la edición alemana comienza con un tomo de salud pública que no se encuentra en la traducción. Continúa con las enfermedades infecciosas y hasta el cuarto volumen no se ocupa de las enfermedades del aparato respiratorio, que es preci- 
samente por donde comienza la traducción al castellano. El aparato respiratorio ocupa dos volúmenes en la versión original y tres en la traducida. Con respecto a las enfermedades infecciosas, ocupan los volúmenes segundo y tercero de la edición original, pero no aparecen en la traducción hasta los tomos 20, 21 y 22, con un retraso, además, de más de diez años sobre la publicación original. Los tomos cuarto a noveno de la traducción se ocupan del sistema nervioso, cuando la edición original no se ocupa de ello hasta su volumen undécimo. Es difícil saber a qué criterios obedeció esta elección: ¿era más relevante para los médicos españoles conocer los avances en torno al sistema respiratorio y al nervioso que lo relativo a las enfermedades infecciosas o al aparato circulatorio? ¿O acaso estaba más preparado el médico-traductor o su equipo para hacer frente a las traducciones de unos sistemas por encima de otros? ¿Se trataba quizá de un interés especial y personal? ¿El clima y situación socioeconómica del país influyó sobre estas decisiones? Son preguntas para las que procuraremos hallar algún indicio de respuesta en el curso de la investigación. Otro interrogante que se plantea es por qué de las enfermedades relativas a los órganos sexuales femeninos, que ocupan todo un volumen en la edición original (el tomo 10), no se halla ni rastro en la traducción. ¿Es acaso un ejemplo de adaptación a la moral de la época? Igualmente sorprende la ausencia en la edición en español de dos grandes ámbitos, correspondientes a los tomos 14 y 16 de la versión original: las enfermedades de la piel y las enfermedades mentales. Por último, no existe en la edición española un volumen final con la nómina de autores ni con el índice de lemas, cosa que sí ocurre en el último volumen de la obra original. Ello se debe a que todos los volúmenes en español recogen en sus páginas finales un índice terminológico de lo incluido en cada volumen.

Respecto al cotejo de los contenidos se destacan en las siguientes páginas los hallazgos más interesantes y representativos de la traducción.

\subsection{Caso de actualización}

En primer lugar se presenta un caso de actualización del contenido de la obra original: en el tomo tercero de la traducción, de 1887 (tomo $\mathrm{V}$ del original [1874]), al final del capítulo titulado «Tuberculosis crónica y aguda», el traductor añade la siguiente nota al pie para justificar la inclusión de un apéndice de 60 páginas (855-915):

Hay que tener en cuenta que han trascurrido más de diez años entre la publicación original y la traducción, de modo que parece muy sensato que el médico-traductor asuma la responsabilidad de incluir nada menos que 60 páginas de cosecha propia sobre los hallazgos más recientes en torno al tratamiento de la tuberculosis.

Hay que añadir que en esas 60 páginas se cita la bibliografía consultada en forma de notas al pie y se detecta cierto equilibrio entre las obras consultadas de origen francés, alemán e inglés/estadounidense.

\subsection{Caso de adaptación}

Por otro lado, al igual que en el caso anterior de Eulenburg, en el campo de las aguas también se hallan adaptaciones locales. Así, en el tercer tomo de la edición española (pp. 914-915) aparece una tabla bajo este anuncio: «Accediendo al deseo manifestado por varios suscritores, ponemos á continuación un cuadro de las aguas minero- 
medicinales extranjeras citadas en las enfermedades del aparato respiratorio de esta obra y las equivalentes de España».

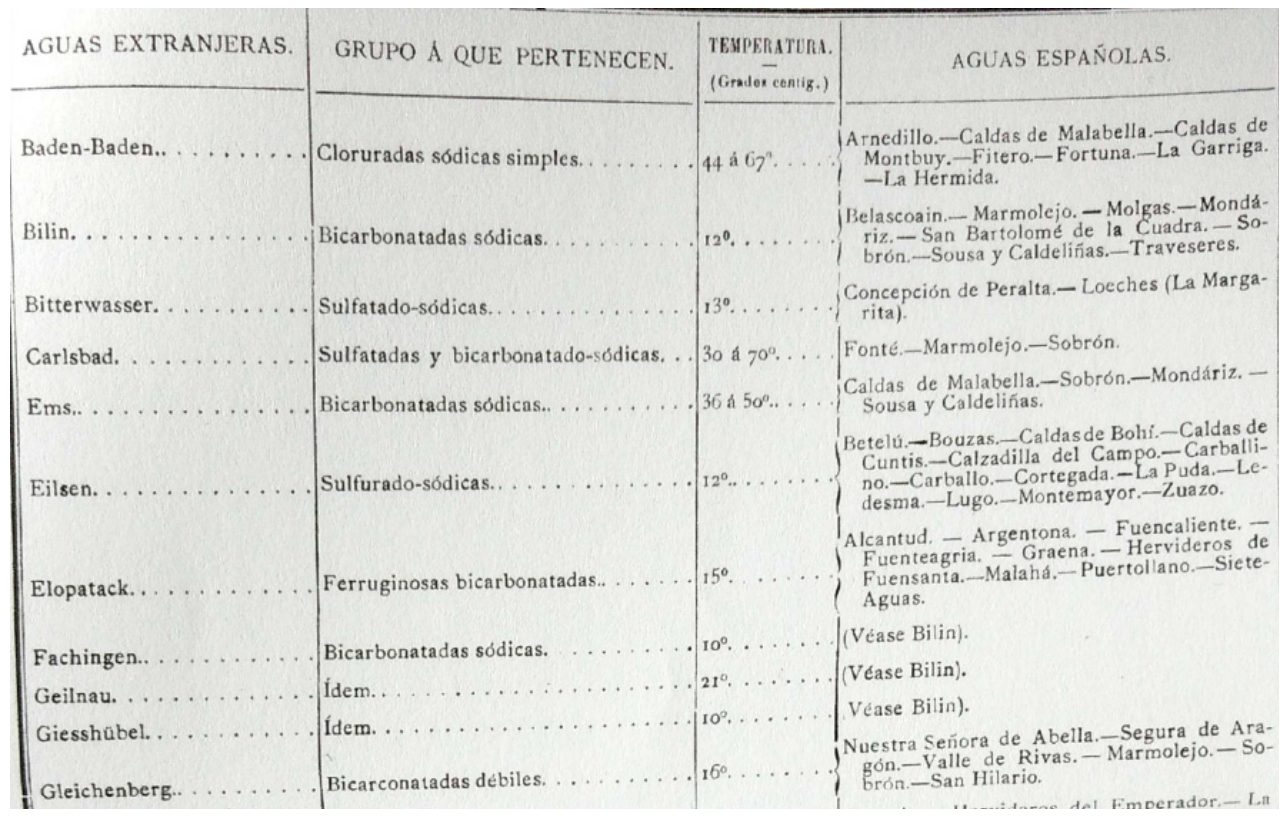

Figura 5: Tabla aparecida en el volumen 3 de la edición en castellano (pp. 914-915).

Este comportamiento es en todo caso desigual a lo largo de la obra traducida, pues en otras partes se mantienen los nombres de ciudades alemanas, como por ejemplo en el tomo 2 de la versión española, cuando se citan las ciudades en las que se recomienda pasar el invierno (tomo 2, p. 141), donde se mencionan las mismas que en la versión original (tomo 4, p. 139), lo cual no parece muy lógico para el lector hispanohablante, que raramente irá a pasar el invierno a las ciudades alemanas que se proponen en la versión original.

En conclusión, sobre este manual de Ziemssen queda aún mucho por hacer, y las observaciones preliminares hacen pensar que el estudio traductológico será especialmente interesante, habida cuenta del número relativamente elevado para la época de notas del traductor a pie de página, algunas sobre insertos para actualizar información, otras sobre comentarios lingüísticos, y otras que se refieren al acortamiento o ampliación de ciertas entradas. Sobre todo ello esperamos poder exponer resultados en próximos trabajos.

\section{CONCLUSIONES PROVISIONALES}

No se pueden extraer conclusiones definitivas de este pequeño análisis, pues son muchas las voces que contienen estos diccionarios y pocas las que se han podido estudiar hasta el momento. No obstante, y retomando lo expuesto anteriormente, cabría decir que en esta traducción y adaptación al español encontramos casos curiosos sobre 
cómo se vierte el contenido original: se da la síntesis, en efecto (en el diccionario de Eulenburg no aparecen en español las ciudades balneario citadas bajo Bad-, se eliminan numerosos párrafos del lema Wasser en la versión en español y se aúnan bajo un lema, resumidos, los contenidos de dos lemas originales); se da también la modificación del contenido (tanto en la bibliografía final, que añade obras consultadas por el médico traductor y nombres de doctores españoles, como en el propio contenido de algunas entradas); se da la adaptación al lector al eliminar las partes referidas a las características de las aguas alemanas y se resume información que no parece pertinente para los médicos españoles; finalmente, se da también, como se ha visto, la ampliación o sustitución del contenido por actualización en cuanto a los hallazgos o por inclusión de informaciones relevantes para los médicos españoles.

Se observa, además, un mimo por el lenguaje y un cuidado de la redacción envidiables y constantes a lo largo de toda la obra traducida en ambos casos, con abundancia también de notas del traductor e incisos aclaratorios que dicen mucho de la ingente labor a la que se hubieron de enfrentar estos médicos traductores y del cariño con que la encararon.

Los resultados obtenidos hasta la fecha no son en modo alguno concluyentes y esperamos poder ofrecer más datos en futuros trabajos. Como se apuntaba al inicio, la versión en español de estas enciclopedias médicas nos puede decir mucho sobre cómo se recibía y también sobre cómo iba evolucionando la medicina en nuestra España decimonónica. La labor de estos traductores y médicos los convierte verdaderamente en copartícipes de la creación del conocimiento en nuestro país y al menos a ellos les debemos el continuar analizando su trabajo y elogiando su buen hacer.

\section{REFERENCIAS BIBLIOGRÁFICAS}

ECKART, Wolfgang U. $\left(2009^{6}\right)$ : Geschichte der Medizin, Heidelberg, Springer.

EULENBURG, Albert (1880-1883): Real-Encyclopädie der gesamten Heilkunde: Medizinisch-chirurgisches Handwörterbuch für praktische Ärzte, Viena y Leipzig, Urban \& Schwarzenberg.

EULENBURG, Albert (1885-1891): Diccionario enciclopédico de medicina y cirugía prácticas, escrito en alemán bajo la dirección del Dr. A. Eulenburg; traducido directamente y arreglado para uso de los médicos españoles por el Dr. D. Isidoro de Miguel y Viguri, Madrid, Agustín Jubera.

GuTIÉRREZ Rodilla, Bertha M. (1999): La constitución de la lexicografia médica moderna en España, Noya, Toxosoutos.

GutiéRREZ RoDiLla, Bertha M. (2009): «Científica, traducción», en Francisco Lafarga y Luis Pegenaute, eds., Diccionario histórico de la traducción en España, Madrid, Gredos, pp. 230-239.

GutiérREz RoDILLA, Bertha M. (2012a): «El Vocabulario terminológico de medicina de Manuel Hurtado de Mendoza», Revista de Filología Española, 92, 2, pp. 249-272. 
GutiÉRrez RodiLla, Bertha M. (2012b): «La obra lexicográfica de Manuel Hurtado de Mendoza: sus diccionarios enciclopédicos de medicina», Asclepio. Revista de Historia de Medicina y de la Ciencia, LXIV, 2, pp. 467-490.

GUTIÉRREZ Rodilla, Bertha M. y Carmen QuIJADA DiEZ (2015): «La adaptación del contenido en los diccionarios médicos traducidos y publicados en España en el siglo XIX», en P. Aullón de Haro y Alfonso Silván, eds., Translatio y Cultura, Madrid, Dykinson, pp. 201-207.

MONTALT I RESURRECCIÓ, Vicent (2002): «Los traductores, partícipes en la construcción del conocimiento científico», en A. Andreu, ed., Conocimiento e invención, Valencia, Universidad Politécnica de Valencia, pp. 516-527.

Montalt i ResurRecció, Vicent (2005): Manual de traducción cientificotécnica, Vic, Eumo Editorial.

OlOHAN, Maeve $\left(2009^{2}\right)$ : «Scientific and technical translation», en Mona Baker y Gabriela Saldanha, eds., Routledge encyclopedia of translation studies, Londres, Routledge, pp. 246-249.

QuiJADA DiEZ, Carmen (2016): «Traducción al español del diccionario médico alemán de Albert Eulenburg», en Ignacio Sariego López, Juan Gutiérrez Cuadrado y Cecilio Garriga Escribano, eds., El diccionario en la encrucijada: de la sintaxis y la cultura al desafio digital, Santander, Escuela Universitaria de Turismo Altamira y Asociación Española de Lexicografía Hispánica, pp. 369-380.

ZIEMSSEN, Hugo von (1874-1885): Handbuch der speciellen Pathologie und Therapie, Leipzig, Verlag von F. C. W. Vogel.

ZIEMSSEN, Hugo von (1887-1898): Tratado enciclopédico de patología médica y terapéutica traducido al español por el Dr. Francisco Vallina, Madrid, Sucesores de Rivadeneyra.

\section{TÍTULO/TITLE}

«La traducción al español de diccionarios médicos alemanes en el siglo XIX» «The translation into Spanish of German medical dictionaries in the 19th century»

Fecha de presentación: 30/10/2017 Fecha de aceptación: 29/01/2018

Resumen: En el presente trabajo se expone el estudio traductológico preliminar llevado a cabo sobre dos diccionarios médicos alemanes del siglo XIX y traducidos al español al final de la centuria, en un momento en que la traducción científica al castellano se ejercía sobre textos procedentes casi siempre de Francia, lo que los convierte en una fuente de singular valía para el estudio de la recepción de la ciencia en la España decimonónica. Se trata de la obra de Albert Eulenburg Real-Encyclopädie der gesamten Heilkunde: Medizinisch-chirurgisches Handwörterbuch für praktische Ärzte (1880-1883), editado en español bajo el título Diccionario enciclopédico de medicina y cirugía prácticas, «traducido directamente y arreglado para uso de los médicos españoles» por Isidoro de Miguel y Viguri (1885-1891) y del Handbuch der speciellen Pathologie und Therapie de Hugo von Ziemssen (18741885), traducido al castellano por Francisco Vallina entre 1887 y 1901 bajo el título Tratado enciclopédico de patología médica y terapéutica. A lo largo del estudio se ha podido comprobar que en la traslación de estos textos al castellano se observan importantes síntesis de contenido, modificaciones para actualizar los conocimientos médicos en el momento inicial de su formulación, ampliación y 
sustitución del contenido original para adaptarlo al público destinatario e incluso corrección de la información original por errónea o incluso antiespañola. Todo ello se presenta aquí con ejemplos extraídos de las obras analizadas.

Palabras clave: diccionarios médicos; traducción alemán-español; recepción de la ciencia; lexicografía médica.

Abstract: In this work we present the preliminary translation study carried out on two medical German dictionaries written in the nineteenth century and translated into Spanish in the last decade of the century, at a time when scientific translation into Spanish took place mainly from French texts, which makes of these analyzed works quite a valuable source to study science reception in nineteenthcentury Spain. They are Albert Eulenburg's Real-Encyclopädie der gesamten Heilkunde: Medizinisch-chirurgisches Handwörterbuch für praktische Ärzte (1880-1883), «directly translated and arranged for the use of Spanish doctors» by Isidoro de Miguel y Viguri (1885-1891) and Hugo von Ziemssen's Handbuch der speciellen Pathologie und Therapie (1874-1885), translated into Spanish by Francisco Vallina between 1887 and 1901 under the title Tratado enciclopédico de patología médica y terapéutica. In that translation and adaptation of medical dictionaries, four types of modification are observed with respect to the original texts: synthesis of content, the updating of such, extension or substitution to adapt it to the target audience and correction of the original information for being wrong or even anti-Spanish. We illustrate these modifications by means of examples from the two analyzed dictionaries.

Key words: medical dictionaries; German-Spanish translation; science reception; medical lexicography. 